\title{
SANS Study on Pulley Effect of Slide-ring Gel
}

\author{
Takeshi Karino $^{\text {a,1,* }}$ Mitsuhiro Shibayama ${ }^{\text {a,1 }}$ Yasushi Okumura ${ }^{\text {b,1 }}$ Kohzo Ito $^{\text {b,1 }}$ \\ ${ }^{a}$ Neutron Science Laboratory, Institute for Solid State Physics, University of Tokyo, Tokai, Ibaraki, 319-1106, Japan \\ ${ }^{\mathrm{b}}$ Graduate School of Frontier Sciences, University of Tokyo, Kashiwa, Chiba 277-8561, Japan
}

\begin{abstract}
We have recently developed a novel kind of gel "slide-ring gel", based on polyrotaxane consisting of thread-like molecules of poly(ethylene glycol) and rings of $\alpha$-cyclodextrin (CD) molecules. The slide-ring gel has mobile crosslinks. In order to improve the unique properties, i.e. "sliding motion" or "pulley effect" of the slide-ring gels and to suppress the self-aggregation tendency of CD molecules, methylated polyrotaxane was synthesized by substituting hydroxyl group with methyl group on CD molecules. The structure of SR gel having methylated CD molecules was investigated by small angle neutron scattering.
\end{abstract}

Key words: slide-ring gel, pulley effect, methylated polyrotaxane

\section{Introduction}

We have developed a novel kind of gel called "slidering (SR) gel" based on the supramolecular architecture[1]. The SR gel was prepared by connecting the $\alpha$-cyclodextrin (CD) molecules on polyrotaxane chain, which consists of poly(ethylene glycol) and $\alpha$ cyclodextrin. The cross-links of the SR gel can move along the polymer chain, while those of a conventional polymer gels are fixed on polymer chains by covalent bond or physical interaction/entaglement. This implies that the spatial inhomogeneities of SR gel are effectively reduced by sliding the cross-links. This effect of mobile cross-links is called "pulley effect". The SR gel with mobile cross-links show high extensibility, large degree of swelling, and large reversible deformability. In previous works, we discussed the static structure factor [2] and deformation mechanism[3] in-

\footnotetext{
* Neutron Science Laboratory, Institute for Solid State Physics University of Tokyo Kashiwanoha 5-1-5, Kashiwa, Chiba 2778581 JAPAN. Phone: +81 4-7136-3424 Fax: +81 4-7134-6069, Email: karino@issp.u-tokyo.ac.jp

1 This work was supported by Core Research for Evolutional Science and Technology (CREST), Japan Science and Technology Agency, Japan.
}

vestigated by small angle neutron scattering (SANS). A 2D SANS pattern of SR gels under uniaxial deformation showed a normal butterfly pattern. Abnormal butterfly patterns have been exclusively observed in polymer gels [4][5][6]. Hence, it was the first time that a normal butterfly pattern was observed in polymer gels by stretching. This result implies that the spatial inhomogeneities can be reduced owing to sliding motion of the cross-links. It was also found that the structure of the SR gels is strongly dependent on the kind of solvents. That is, the polyrotaxane chains, i.e., the pregel solutions, behave like Gaussian chains in a NaODaq because CD molecules are molecularly dispersed due to the repulsive electrostatic interaction of dissociated hydroxyl groups on CD. On the other hand, the polyrotaxane chains become rigid in dimethylsulfoxide (DMSO) due to hydrogen bonding of neighboring CD molecules on a chain. It should be noted here that a solvent capable of breaking inter-molecular hydrogen bond is necessary to dissolve the polyrotaxane. Hence, we employed NaOHaq and DMSO as a solvent of the polyrotaxane carring CD molecules.

The polyrotaxane is soluble exclusibely in DMSO and NaOHaq. The other solvent, such as organic solvent and water did not dissolve polyrotaxane as is the case of cellulose. This fact is a serious problem when 
one applies the SR gel to nanotechnology. Recently, Araki et al reported the solubility of polyrotaxane in dimethylacetamide/lithium chloride system [7].

We synthesized methylated polyrotaxane where the hydroxyl groups on CD were substituted by methyl groups. Interestingly, the methylated polyrotaxane was soluble in water. In the present paper, we discuss how this type of chemical modification influences the pulley effect.

\section{Experimental}

Polyrotaxane was prepared with poly(ethylene glycol) (PEG) and $\alpha$-cyclodextrin (CD). The molecular weight of PEG was $3.5 \times 10^{4}$. The polydispersity of PEG was 1.06. The filling ratio of CD was $27 \%$. The details of sample preparation are described elsewhere[2] [8]. The hydroxyl groups on each CD molecule were substituted by methyl groups using sodium hydrate and methyl iodide. The degree of methylation, $f_{\mathrm{m}}$, was controlled by the feed of reaction regent. In this study, $f_{\mathrm{m}}$ was chosen to be $20,60,80 \%$. The sample codes were defined by M20, M60, M80.

The SR gels were prepared in $1 \mathrm{~N} \mathrm{NaODaq}$, where divinyl sulfone was used as cross-linker. The polymer concentration was $10 \mathrm{wt} \%$. The cross-linker concentration was $2 \mathrm{wt} \%$

Small-angle neutron scattering (SANS) experiments were carried out at the SANS-U spectrometer at Institute for Solid State Physics, University of Tokyo, Tokai, Japan. Two-dimensional scattering intensity patterns were collected with a two-dimensional detector placed at $8 \mathrm{~m}$ and $2 \mathrm{~m}$ positions from the samples. The incident neutron wavelength was $7.0 \AA$. The temperature of the samples was regulated to be $20^{\circ} \mathrm{C}$ with a watercirculating bath controlled with a Neslab RTE-111 thermocontroller with a precision of $\pm 0.1^{\circ} \mathrm{C}$. The observed scattered intensity functions were corrected for air scattering, incoherent scattering, and transmission and then were rescaled to the absolute intensity with a polyethylene (Lupolen) secondary standard[9].

\section{Result and Discussion}

Fig. 1 shows the Kratky plot of SR gels with various $f_{\mathrm{m}}$ 's, where $I(q)$ is the scattering intensity and $q$ is the momentum transfer. In the case of M20, the function is characterized by a peak appearance at $0.02 \AA^{-1}$. Such an appearance of maximum in a Kratky plot is commonly observed in polymer gels, which is an indication of the presence of frozen inhomogeneities. In the case of $\mathrm{M} 60$, on the other hand, $q^{2} I(q)$ changed to a monoton-

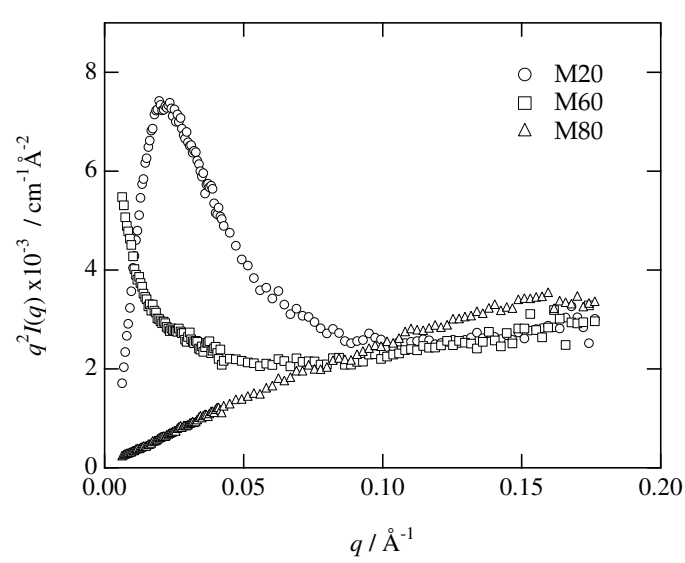

Fig. 1. The Kratky plot of SR gel with various $f_{\mathrm{m}}$ 's.

ically decreasing function with $q$. In M80, even an upturn at the low- $q$ region disappeared and $q^{2} I(q)$ looked like that of a polymer solution. The scattering intensity function for as-prepared polymer gels is given by a sum of Lorentz and squared-Lorentz functions [10]. Lorentz function is so-called Ornstein-Zernike function which describes a polymer concentration fluctuation. On the other hand, Squared-Lorentz function describes an excess scattering from spatial inhomogeneities.

$I(q)=\frac{I_{\mathrm{SL}}(0)}{\left(1+q^{2} \Xi^{2}\right)^{2}}+\frac{I_{\mathrm{L}}(0)}{\left(1+q^{2} \xi^{2}\right)}$

where $I_{\mathrm{L}}(0)$ and $I_{\mathrm{SL}}(0)$ are the zero- $q$ intensities corresponding to the susceptibility and spatial inhomogeneities, respectively. $\xi$ is the correlation length and $\Xi$ is the characteristic length representing inhomogeneities. For M60 and M80, we analyzed the scattering function by using Eq. 1. The obtained values were $\Xi=222.0 \AA$ and $108.7 \AA$ and $\xi=130.9 \AA$ and $23.9 \AA$, respectively for M60 and M80. We also obtained the following values, $996.7 \mathrm{~cm}^{-1}$ and $8.8 \mathrm{~cm}^{-1}$ for $I_{\mathrm{SL}}(0)$, $40.3 \mathrm{~cm}^{-1}$ and $1.34 \mathrm{~cm}^{-1}$ for $I_{\mathrm{L}}(0)$. These results indicats that the degree of spatial inhomogeneities of M80 is much lower than that of M60.

CD molecules on the polymer chain have a tendency to aggregate due to hydrogen bonding between hydroxyl groups on the CD molecules. Because of this, not only the structure of SR gel but also the solubility of polyrotaxane in a solvent is strongly affected by the aggregation state of CD molecules. In NaODaq, hydroxyl groups of CD molecules are dissociated. By introducing charge groups in gels, the scattering intensity becomes lower than that of non-charged gels. This is due to suppression of concentration fluctuations. In this case, since the number of hydroxyl group decreases with increasing $f_{\mathrm{m}}$, the scattering intensity of M20 is expected to be lower than those of the others. However, the obtained scattering intensity is higher than the oth- 
ers. This means that in M20, the inhomogeneities are not effectively reduced by the dissociatioated groups. However, it is interesting to note that the scattering intensity decreased with increasing $f_{\mathrm{m}}$. This indicates that the inhomogeneities were effectively suppressed by substituting hydroxyl groups with methyl groups. The CD molecules on the polymer are dispersed molecularly due to inhibition of hydrogen-bonding cluster formation between $\mathrm{CD}$ molecules. In the low $f_{\mathrm{m}}$, the competition between dissociated hydroxyl groups and non-charged methyl groups leads to an increase of inhomogeneities in polymer gel, resulting the higher scattering intensity as was observed in M20. These result suggests that the sliding motion can be controlled by varying the $f_{\mathrm{m}}$.

\section{Conclusion}

The methylated polyrotaxane was synthesized by substituting the hydroxyl groups of CD molecules on PEG chains. The scattering intensity of gels decreased with increasing the methylation degree. By substituting, the hydrogen-bonding formation between CD molecules was suppressed. These facts suggests that the inhomogeneities in gels are suppressed by an improvement of sliding mobility of the CD molecules. It is concluded that the sliding motion of SR gel can be controlled by chemical modification of the CD molecules of the SR gels.

\section{Acknowledgment}

This work is supported by Core Research for Evolutional Science and Technology (CREST), Japan Science and Technology Agency, Japan. This work was also partially supported by the Ministry of Education, Science, Sports and Culture, Japan (Grant-inAid, 14045216 and 16350120). The SANS experiment was performed with the approval of Institute for Solid State Physics, The University of Tokyo (Proposal Nos. 02- 2529, 03-3510), at Japan Atomic Energy Research Institute, Tokai, Japan.

\section{References}

[1] Y. Okumura, K. Ito, Adv. Mater. 13 (2001) 485.

[2] T. Karino, Y. Okumura, K. Ito, M. Shibayama, Macromolecules 37 (2004) 6177.

[3] T. Karino, Y. Okumura, C. Zhao, T. Kataoka, K. Ito, M. Shibayama, Macromolecules 38 (2005) 6161.

[4] E. J. Mendes, P. Lindner, M. Buzier, F. Boué, J. Bastide, Phys. Rev. Lett. 66 (1991) 1595.
[5] A. Ramzi, A. Hakiki, J. Bastide, F. Boué, Macromolecules 30 (1997) 2963.

[6] M. Shibayama, K. Kawakubo, F. Ikkai, M. Imai, Macromolecules 31 (1998) 2586.

[7] J. Araki, K. Ito, J. Polym. Sci. A 44 (2006) 532.

[8] J. Araki, C, Zhao, K. Ito, Macromolecules 38 (2005) 7524.

[9] M. Shibayama, M. Nagao, S. Okabe, T. Karino, J. Phys. Soc. Jpn. 74 (2005) 2728.

[10] M.Shibayama, K. Isono, S. Okabe, T. Karino, M. Nagao, Macromolecules, 37 (2004) 2909. 